TRANSACTIONS OF THE

AMERICAN MATHEMATICAL SOCIETY

Volume 356, Number 8 , Pages 3005-3023

S 0002-9947(03)03486-X

Article electronically published on December 9, 2003

\title{
A NEW VARIATIONAL CHARACTERIZATION OF $n$-DIMENSIONAL SPACE FORMS
}

\author{
ZEJUN HU AND HAIZHONG LI
}

\begin{abstract}
A Riemannian manifold $\left(M^{n}, g\right)$ is associated with a Schouten $(0,2)$-tensor $C_{g}$ which is a naturally defined Codazzi tensor in case $\left(M^{n}, g\right)$ is a locally conformally flat Riemannian manifold. In this paper, we study the Riemannian functional $\mathcal{F}_{k}[g]=\int_{M} \sigma_{k}\left(C_{g}\right) d v o l_{g}$ defined on $\mathcal{M}_{1}=\{g \in$ $\mathcal{M} \mid \operatorname{Vol}(g)=1\}$, where $\mathcal{M}$ is the space of smooth Riemannian metrics on a compact smooth manifold $M$ and $\left\{\sigma_{k}\left(C_{g}\right), 1 \leq k \leq n\right\}$ is the elementary symmetric functions of the eigenvalues of $C_{g}$ with respect to $g$. We prove that if $n \geq 5$ and a conformally flat metric $g$ is a critical point of $\left.\mathcal{F}_{2}\right|_{\mathcal{M}_{1}}$ with $\mathcal{F}_{2}[g] \geq 0$, then $g$ must have constant sectional curvature. This is a generalization of Gursky and Viaclovsky's very recent theorem that the critical point of $\left.\mathcal{F}_{2}\right|_{\mathcal{M}_{1}}$ with $\mathcal{F}_{2}[g] \geq 0$ characterized the three-dimensional space forms.
\end{abstract}

\section{INTRODUCTION}

Let $M^{n}$ be an $n$-dimensional compact and smooth manifold. Denote by $\mathcal{M}$ and $\mathcal{G}$ the space of smooth Riemannian metrics and the diffeomorphism group of $M$, respectively. We call a functional $\mathcal{F}: \mathcal{M} \rightarrow R$ Riemannian if $\mathcal{F}$ is invariant under the action of $\mathcal{G}$, i.e., $\mathcal{F}\left(\varphi^{*} g\right)=\mathcal{F}(g)$ for each $\varphi \in \mathcal{G}$ and $g \in \mathcal{M}$.

By letting $S_{2}(M)$ denote the bundle of symmetric $(0,2)$-tensors on $M^{n}$, we say that $\mathcal{F}: \mathcal{M} \rightarrow R$ has a gradient at $g \in \mathcal{M}$ if

$$
\left.\frac{d}{d t} \mathcal{F}(g+t h)\right|_{t=0}=\int_{M}\langle h, \nabla \mathcal{F}\rangle_{g} d V o l_{g}
$$

for some $\nabla \mathcal{F} \in \Gamma\left(S_{2}(M)\right)$ and all $h \in \Gamma\left(S_{2}(M)\right)$. The theory of Riemannian functionals has a long history; for details and references we refer to [1] and [4, among many others.

Following [4] and [10], we consider the functional

$$
\mathcal{F}_{k}[g]=\int_{M^{n}} \sigma_{k}\left(C_{g}\right) d V o l_{g},
$$

Received by the editors September 30, 2002.

2000 Mathematics Subject Classification. Primary 53C20, 53C25.

Key words and phrases. Locally conformally flat Riemannian manifold, Schouten tensor, space form, Riemannian functional.

The first author was partially supported by grants from CSC, NSFC and the Outstanding Youth Foundation of Henan, China.

The second author was partially supported by the Alexander von Humboldt Stiftung and Zhongdian grant of NSFC. 
where $\sigma_{k}\left(C_{g}\right)$ is the $k$-th elementary symmetric function of the eigenvalues of the Schouten tensor $C_{g}:=\operatorname{Ric}-\frac{r}{2(n-1)} g$ with respect to $g$. Here Ric and $r$ denote the Ricci tensor and the scalar curvature of $g$, respectively. We note that in classical terms the Schouten tensor is $\frac{1}{n-2} C_{g}$.

Recall that an $n$-dimensional Riemannian manifold $\left(M^{n}, g\right)$ is said to be locally conformally flat if it admits a coordinate covering $\left\{U_{\alpha}, \varphi_{\alpha}\right\}$ such that the map $f_{\alpha}:\left(U_{\alpha}, g_{\alpha}\right) \rightarrow\left(S^{n}, g_{0}\right)$ is a conformal map, where $g_{0}$ is the standard metric on $S^{n}$. Since a 2-dimensional Riemannian manifold is always locally conformally flat, we will assume $n \geq 3$ throughout this paper.

In [10] Viaclovsky studied $\left.\mathcal{F}_{k}\right|_{[g]_{1}}$, where $g$ is a fixed smooth metric on $M^{n}$ and $[g]_{1}$ denotes the space of smooth metrics which are conformal to $g$ and have unit volume, and he proved that a metric $\tilde{g}$ is critical for $\left.\mathcal{F}_{k}\right|_{[g]_{1}}$ if and only if $\sigma_{k}\left(C_{\tilde{g}}\right)=$ constant provided $k=1,2$; or $k \geq 3$ and $\left(M^{n}, g\right)$ is locally conformally flat. Let $\mathcal{M}_{1}=\{g \in \mathcal{M} \mid \operatorname{Vol}(g)=1\}$. In [4] the authors considered $\mathcal{F}_{2}[g]$ on $\mathcal{M}_{1}$ and proved the following important result.

Theorem A (4]). Let $M$ be compact and three-dimensional. Then a metric $g$ with $\mathcal{F}_{2}[g] \geq 0$ is critical for $\left.\mathcal{F}_{2}\right|_{\mathcal{M}_{1}}$ if and only if $g$ has constant sectional curvature.

Our main purpose in this paper is to generalize Theorem A to a higher-dimensional situation. This is achieved when we restrict ourselves to the case that the critical metric is locally conformally flat. Precisely, our main result is the following

Theorem B. Let $M^{n}$ be compact with dimension $n \geq 5$. Then a conformally flat metric $g$ with $\mathcal{F}_{2}[g] \geq 0$ is critical for $\left.\mathcal{F}_{2}\right|_{\mathcal{M}_{1}}$ if and only if $g$ has constant sectional curvature.

Remark 1.1. Similar to the $n=3$ case in Theorem A, the condition $\mathcal{F}_{2}[g] \geq 0$ in Theorem B remains necessary: Let $E=R i c-\frac{r}{n} g$ denote the trace-free Ricci tensor; then

$$
\sigma_{2}\left(C_{g}\right)=-\frac{1}{2}|E|^{2}+\frac{(n-2)^{2}}{8 n(n-1)} r^{2} .
$$

If $g$ has constant sectional curvature, then $E=0$ and $\sigma_{2}\left(C_{g}\right)=(n-2)^{2} r^{2} /$ $[8 n(n-1)] \geq 0$. However, there do exist critical metrics with $\mathcal{F}_{2}<0$; see Remark 7.1 below.

In this paper, all manifolds are supposed to be smooth, connected and orientable for compact ones.

\section{Preliminaries}

Let $\left(M^{n}, g\right)$ be an $n$-dimensional Riemannian manifold. We choose a local orthonormal vector field $\left\{e_{1}, \cdots, e_{n}\right\}$ adapted to the Riemannian metric of $\left(M^{n}, g\right)$ with $\left\{\omega_{1}, \cdots, \omega_{n}\right\}$ its dual coframe. Then the connection forms $\left\{\omega_{i j}\right\}$ of $\left(M^{n}, g\right)$ are characterized by the structure equations

$$
\begin{aligned}
d \omega_{i} & =-\sum_{j} \omega_{i j} \wedge \omega_{j}, \quad \omega_{i j}+\omega_{j i}=0, \\
d \omega_{i j} & =-\sum_{k} \omega_{i k} \wedge \omega_{k j}+\frac{1}{2} \sum_{k, l} R_{i j k l} \omega_{k} \wedge \omega_{l},
\end{aligned}
$$


where $R_{i j k l}$ are the components of the Riemannian curvature tensor of $\left(M^{n}, g\right)$. Let $W_{i j k l}$ denote the components of the Weyl curvature tensor of $\left(M^{n}, g\right)$, i.e. (see [1]),

$$
W_{i j k l}=R_{i j k l}-\frac{1}{n-2}\left(C_{i k} g_{j l}-C_{i l} g_{j k}+C_{j l} g_{i k}-C_{j k} g_{i l}\right),
$$

where $C$ is the so-called Schouten tensor and it is a symmetric $(0,2)$-tensor defined by

$$
C=R i c-\frac{r}{2(n-1)} g
$$

with Ric and $r$ denoting the Ricci curvature tensor and scalar curvature of $g$, respectively. In the sequel we often write $C$ as $C_{g}$ in emphasizing its dependence on the metric $g$.

Let $R_{i j}$ be the components of Ric; then $C_{i j}=R_{i j}-\frac{r}{2(n-1)} g_{i j}$. Denote by $\nabla$ the covariant derivative on $\left(M^{n}, g\right)$ and write, e.g., $R_{i j, k}=\nabla_{k} R_{i j}, R_{i j, k l}=\nabla_{l} \nabla_{k} R_{i j}$, $C_{i j, k}=\nabla_{k} C_{i j}, C_{i j, k l}=\nabla_{l} \nabla_{k} C_{i j}$, and so on. Then we have the following Ricci identities (cf. [5]):

$$
\begin{aligned}
& R_{i j, k l}-R_{i j, l k}=\sum_{m} R_{m j} R_{m i k l}+\sum_{m} R_{i m} R_{m j k l}, \\
& C_{i j, k l}-C_{i j, l k}=\sum_{m} C_{m j} R_{m i k l}+\sum_{m} C_{i m} R_{m j k l} .
\end{aligned}
$$

Let $B$ denote the Cotten tensor, i.e., $B_{i j k}=C_{i j, k}-C_{i k, j}$. Now, we can state the following well-known facts:

(i) If $n=3$, we always have $W_{i j k l} \equiv 0 .\left(M^{3}, g\right)$ is locally conformally flat if and only if $B_{i j k} \equiv 0$.

(ii) If $n \geq 4,\left(M^{n}, g\right)$ is locally conformally flat if and only if $W_{i j k l} \equiv 0$.

(iii) $\sum_{i} W_{i j k l, i}=\frac{n-3}{n-2} B_{j k l}$, and so $B_{i j k} \equiv 0$ provided $\left(M^{n}, g\right)$ is locally conformally flat and $n \geq 4$.

Hence, if $\left(M^{n}, g\right)$ is a locally conformally flat manifold with $n \geq 3$, then we have

$$
\begin{gathered}
R_{i j k l}=\frac{1}{n-2}\left(R_{i k} g_{j l}-R_{i l} g_{j k}+R_{j l} g_{i k}-R_{j k} g_{i l}\right)-\frac{r}{(n-1)(n-2)}\left(g_{i k} g_{j l}-g_{i l} g_{j k}\right), \\
C_{i j, k}=C_{i k, j} ;
\end{gathered}
$$

the latter means that $C_{i j}$ is a Codazzi tensor.

Let $\tilde{C}_{i}^{j}=C_{i k} g^{k j}$. Then we define a family of invariant functions $\sigma_{k}\left(C_{g}\right)$ of $\left(M^{n}, g\right)$ by

$$
\operatorname{det}(\tilde{C}+t I):=\operatorname{det}\left(\tilde{C}_{i}^{j}+t \delta_{i}^{j}\right)=\sum_{k=0}^{n} \sigma_{k}\left(C_{g}\right) t^{n-k},
$$

i.e., $\sigma_{k}\left(C_{g}\right)$ are the $k$-th elementary symmetric functions of the eigenvalues of the tensor $C_{g}$ with respect to $g$ for $1 \leq k \leq n$, and $\sigma_{0}\left(C_{g}\right)=1$. Here the $(1,1)$-tensor $\tilde{C}$ is considered as an endomorphism on $T M$ and $I$ denotes both the unit matrix and the identity endomorphism on $T M$.

Notice that by considering the elementary symmetric functions on $R^{n}$,

$$
S_{k}\left(x_{1}, \cdots, x_{n}\right)=\sum_{1 \leq i_{1}<\cdots<i_{k} \leq n} x_{i_{1}} \cdots x_{i_{k}}, \quad 1 \leq k \leq n ; \quad S_{0}:=1,
$$


we then have

$$
\sigma_{k}\left(C_{g}\right)=S_{k}\left(\lambda_{1}, \cdots, \lambda_{n}\right), \quad 0 \leq k \leq n,
$$

where the $\lambda_{i}$ 's are the eigenvalues of $C_{g}$ with respect to $g$.

By the $(1,1)$-tensor $\tilde{C}$, we can define a series of new endomorphism fields $\left\{T_{k}\right\}_{0 \leq k \leq n-1}$ on $T M$, the so-called Newtonian transformations, by

$$
\operatorname{det}(\tilde{C}+t I) \cdot(\tilde{C}+t I)^{-1}=\sum_{k=0}^{n-1} T_{k}(C) t^{n-k-1},
$$

in the subset $\Omega_{1}$ of $M \times R$ with $\Omega_{1}:=\{(p, t) \mid \operatorname{det}(\tilde{C}(p)+t I(p)) \neq 0\}$.

From (2.9) and (2.12), one can derive the formula

$$
T_{k}=\sigma_{k} I-\sigma_{k-1} \tilde{C}+\cdots+(-1)^{k} \tilde{C}^{k}, \quad k=0,1, \cdots, n-1,
$$

where $\tilde{C}^{k}=\tilde{C} \cdot \tilde{C} \cdots \tilde{C}(k$-tuples $), \sigma_{k}=\sigma_{k}\left(C_{g}\right)$.

\section{Euler-Lagrange equation of $\left.\mathcal{F}_{k}\right|_{\mathcal{M}_{1}}$}

We will need the following properties of the Newtonian transformations $\left\{T_{k}\right\}_{0 \leq k \leq n-1}$ as defined in section two.

Lemma 3.1 (see [4], 8], 9] and 11]). For any $n \times n$ matrix $C=\left(C_{i}^{j}\right)$, we define $\sigma_{k}(C)$ and $T_{k}(C)$ as the $k$-th elementary symmetric polynomials of eigenvalues and the Newtonians of $C$, respectively. Then we have

(1) $\quad(k+1) \sigma_{k+1}=\operatorname{tr}\left(C \cdot T_{k}\right), \quad k=0,1, \cdots, n-1$.

(2) $\left(T_{k}\right)_{i}^{j}=(k !)^{-1} \sum_{i_{1}, \cdots, i_{k} ; j_{1}, \cdots, j_{k}} \delta_{i_{1} \cdots i_{k} i}^{j_{1} \cdots j_{k} j} C_{j_{1}}^{i_{1}} \cdots C_{j_{k}}^{i_{k}}$,

where $\delta_{i_{1} \cdots i_{m}}^{j_{1} \cdots j_{m}}$ is the usual generalized Kronecker symbol, i.e., $\delta_{i_{1} \cdots i_{m}}^{j_{1} \cdots j_{m}}$ equals +1 (resp. - 1$)$ if $\left(j_{1} \cdots j_{m}\right)$ is an even (resp. odd) permutation of $\left(i_{1} \cdots i_{m}\right)$ and in other cases it equals zero.

(3) If the matrix $C=C(t)$ depends smoothly on a real variable $t \in R$, then the corresponding $\sigma_{k}=\sigma_{k}(C(t)), T_{k}=T_{k}(C(t))$ satisfy

$$
\frac{d}{d t} \sigma_{k+1}=\operatorname{tr}\left(\frac{d C}{d t} \cdot T_{k}\right), \quad k=0,1, \cdots, n-1 .
$$

(4) If $C$ is a Codazzi tensor of $(1,1)$-type on a Riemannian manifold $\left(M^{n}, g\right)$, then the corresponding Newtonians have vanishing divergence, i.e.,

$$
\sum_{j}\left[\left(T_{k}\right)_{i}^{j}\right]_{, j}=0, \quad \forall i, k .
$$

(5) If $C=C_{g}$ is given by (2.4) on a Riemannian manifold $\left(M^{n}, g\right)$, then the Newtonian $T_{1}\left(C_{g}\right)$ has vanishing divergence.

Remark 3.1. Property (5), a fact observed in [4] for $n=3$, follows directly from the second Bianchi identity. 
Proposition 3.1. At any $g \in \mathcal{M}$, the functional $\mathcal{F}_{k}[g]=\int_{M} \sigma_{k}\left(C_{g}\right) d V o l_{g}$ defined on $\mathcal{M}$ has a gradient $\nabla \mathcal{F}_{k} \in \Gamma\left(S_{2}(M)\right)$ with the local expression $\nabla \mathcal{F}_{k}=$ $\sum_{i, j}\left(\nabla \mathcal{F}_{k}\right)_{i j} \omega_{i} \otimes \omega_{j}$, where

$$
\begin{aligned}
\left(\nabla \mathcal{F}_{k}\right)_{i j}=- & \frac{1}{2} \Delta_{g}\left(T_{k-1}\right)_{i j}-\sum_{m, l}\left(T_{k-1}\right)^{m l} W_{m i l j}+\frac{r}{n(n-1)}\left(T_{k-1}\right)_{i j} \\
& +\sum_{l}\left[\left(T_{k-1}\right)_{i}^{l}\right]_{, l j}+\frac{2}{n-2} \sum_{l}\left(T_{k-1}\right)_{j}^{l} E_{i l} \\
& -\frac{n}{2(n-1)(n-2)} \operatorname{tr}_{g} T_{k-1} \cdot E_{i j}+\frac{n-2 k-2}{2(n-2)} \sigma_{k} g_{i j} \\
& +\frac{1}{2(n-1)} \Delta_{g}\left(\operatorname{tr}_{g} T_{k-1}\right) \cdot g_{i j}-\frac{1}{2(n-1)}\left(\operatorname{tr}_{g} T_{k-1}\right)_{, i j} \\
& -\frac{1}{2} \sum_{m, l}\left[\left(T_{k-1}\right)^{m l}\right]_{, m l} g_{i j},
\end{aligned}
$$

and where the quantities are all defined by the metric tensor $g, T_{k-1}=T_{k-1}\left(C_{g}\right)$, $\sigma_{k}=\sigma_{k}\left(C_{g}\right), E_{i j}=R_{i j}-\frac{r}{n} g_{i j}$. As usual we use the metric tensor $g$ to raise and lower indices.

Proof. This is a direct calculation, for the convenience of the readers, we conclude it here. We choose a local smooth frame field $\left\{e_{i}\right\}$ with dual $\left\{\omega_{i}\right\}$ on $M^{n}$. Let $g \in \mathcal{M}$ be an arbitrary fixed metric with local expression $g=\sum_{i, j} g_{i j} \omega_{i} \otimes \omega_{j}$. Set $\left(g^{i j}\right)=\left(g_{i j}\right)^{-1}$.

Consider a smooth variation $\tilde{g}(t)$ of $g$ with $\tilde{g}(0)=g$ and $\tilde{g}(t)=\sum_{i, j} \tilde{g}_{i j}(t) \omega_{i} \otimes \omega_{j}$. Let $h_{i j}:=\delta \tilde{g}_{i j}$, where and later in this section $\delta:=\left.\frac{d}{d t}\right|_{t=0}$. As has been explained in Proposition 3.1, we will use $g_{i j}$ or $g^{i j}$ to lower or raise indices, e.g., $h^{i j}:=g^{i k} h_{k l} g^{l j}$. The covariant derivation is with respect to the fixed metric $g$. Then we have the following formulas (cf. [1], [4], [6]):

$$
\begin{aligned}
\delta \tilde{g}_{i j} & =h_{i j}, \quad \delta \tilde{g}^{i j}=-h^{i j}, \\
\delta \tilde{\Gamma}_{i j k} & =\frac{1}{2}\left(h_{j k, i}+h_{i k, j}-h_{i j, k}\right)+\sum_{l} h_{k l} \Gamma_{i j}^{l}, \\
\delta \tilde{\Gamma}_{i j}^{k} & =\frac{1}{2}\left(\nabla_{i} h_{j}^{k}+\nabla_{j} h_{i}^{k}-\nabla^{k} h_{i j}\right),
\end{aligned}
$$

where $\Gamma_{i j k}=\frac{1}{2}\left(\partial_{i} g_{j k}+\partial_{j} g_{i k}-\partial_{k} g_{i j}\right), \quad \Gamma_{i j}^{l}=\sum_{k} g^{k l} \Gamma_{i j k}$ denote the Christoffel symbols w.r.t. the metric $g$; analogously the $\tilde{*}$ - notations correspond to the metric $\tilde{g}_{i j}$.

Then from the expression of the Riemannian curvature tensor

$$
R_{j k l}^{i}=\partial_{k} \Gamma_{j l}^{i}-\partial_{l} \Gamma_{j k}^{i}+\sum_{m}\left(\Gamma_{m k}^{i} \Gamma_{j l}^{m}-\Gamma_{m l}^{i} \Gamma_{j k}^{m}\right), \quad R_{i j k l}=\sum_{m} g_{i m} R_{j k l}^{m},
$$

by a direct calculation we have

$$
\delta \tilde{R}_{i j k l}=\sum_{m} h_{i m} R_{j k l}^{m}+\frac{1}{2}\left(h_{i l, j k}+h_{i j, l k}-h_{j l, i k}-h_{i k, j l}-h_{i j, k l}+h_{j k, i l}\right) .
$$


From $R_{j l}=\sum_{i, k} g^{i k} R_{i j k l}$, we have

$$
\begin{aligned}
\delta \tilde{R}_{j l}= & \frac{1}{2} \sum_{k}\left(h_{l}^{k}\right)_{, j k}+\frac{1}{2} \sum_{k}\left(h_{j}^{k}\right)_{, l k}-\frac{1}{2} \Delta_{g} h_{j l}-\frac{1}{2}\left(\operatorname{tr}_{g} h\right)_{, j l} \\
= & -\frac{1}{2} \Delta_{g} h_{j l}-\frac{1}{2}\left(\operatorname{tr}_{g} h\right)_{, j l}-\sum_{k, m} h^{m k} R_{j m l k} \\
& +\frac{1}{2} \sum_{m}\left[h_{m l} R_{j}^{m}+h_{j m} R_{l}^{m}+\left(h_{l}^{m}\right)_{, m j}+\left(h_{j}^{m}\right)_{, m l}\right],
\end{aligned}
$$

where we have used the Ricci identity

$$
\sum_{k}\left(h_{l}^{k}\right)_{, j k}=\sum_{k}\left(h_{l}^{k}\right)_{, k j}+\sum_{k, m} h^{m k} R_{m l j k}+\sum_{m} h_{m l} R_{j}^{m} .
$$

From $r=\sum_{i, j} g^{i j} R_{i j}, R_{i}^{j}=\sum_{l} g^{j l} R_{i l}$ and (3.2), (3.7), we easily get

$$
\begin{aligned}
\delta \tilde{R}_{i}^{j} & =-\sum_{l} h^{j l} R_{i l}-\frac{1}{2} \Delta_{g} h_{i}^{j}-\frac{1}{2} \sum_{l} g^{j l}\left(\operatorname{tr}_{g} h\right)_{, i l}-\sum_{k, m, l} g^{j l} h^{m k} R_{i m l k} \\
& +\frac{1}{2} \sum_{m}\left[h_{m i} R^{m j}+h_{m}^{j} R_{i}^{m}+\sum_{l} g^{j l}\left(h_{i}^{m}\right)_{, m l}+\left(h^{j m}\right)_{, m i}+\sum_{l} g^{j l}\left(h_{i}^{m}\right)_{, m l}\right] .
\end{aligned}
$$

Combining (3.9) and (3.10) we have

$$
\begin{aligned}
\delta \tilde{C}_{i}^{j}= & \delta \tilde{R}_{i}^{j}-\frac{\delta \tilde{r}}{2(n-1)} \delta_{i}^{j} \\
= & -\sum_{l} h^{j l} R_{i l}-\frac{1}{2} \Delta_{g} h_{i}^{j}-\frac{1}{2} \sum_{l} g^{j l}\left(\operatorname{tr}_{g} h\right)_{, i l}-\sum_{k, m, l} g^{j l} h^{m k} R_{i m l k} \\
& +\frac{1}{2} \sum_{m}\left[h_{m i} R^{m j}+h_{m}^{j} R_{i}^{m}+\sum_{l} g^{j l}\left(h_{i}^{m}\right)_{, m l}+\left(h^{m j}\right)_{, m i}+\sum_{l} g^{j l}\left(h_{i}^{m}\right)_{, m l}\right] \\
& -\frac{1}{2(n-1)} \delta_{i}^{j}\left[-\sum_{m, l} h^{m l} R_{m l}+\sum_{m, l}\left(h^{m l}\right)_{, m l}-\Delta_{g}\left(\operatorname{tr}_{g} h\right)\right] .
\end{aligned}
$$


Therefore, by using Lemma 3.1(3) and (3.11), we obtain

$$
\begin{aligned}
\delta \sigma_{k}\left(C_{\tilde{g}}\right)= & \sum_{i, j}\left(T_{k-1}\right)_{j}^{i} \delta \tilde{C}_{i}^{j} \\
= & -\sum_{i, j, l}\left(T_{k-1}\right)_{j}^{i} h^{j l} R_{i l}-\sum_{i, j, m, l}\left(T_{k-1}\right)^{i j} h^{m l} R_{i m j l} \\
& -\frac{1}{2} \sum_{i, j}\left[\left(T_{k-1}\right)_{j}^{i} \Delta_{g} h_{i}^{j}+\left(T_{k-1}\right)^{i j}\left(\operatorname{tr}_{g} h\right)_{, i j}\right] \\
& +\frac{1}{2} \sum_{i, j, m}\left(T_{k-1}\right)_{j}^{i}\left[h_{m i} R^{m j}+h_{m}^{j} R_{i}^{m}+\left(h^{m j}\right)_{, m i}+\sum_{l} g^{j l}\left(h_{i}^{m}\right)_{, m l}\right] \\
& -\frac{1}{2(n-1)} \operatorname{tr}\left(T_{k-1}\right)\left[-\sum_{i, j} h^{i j} R_{i j}+\sum_{i, j}\left(h^{i j}\right)_{, i j}-\Delta_{g}\left(\operatorname{tr}_{g} h\right)\right] .
\end{aligned}
$$

Note that $\delta \sqrt{\operatorname{det}\left(\tilde{g}_{i j}\right)}=\frac{1}{2} \operatorname{tr}_{g} h \sqrt{\operatorname{det}\left(g_{i j}\right)}$ and therefore $\delta d V o l_{\tilde{g}}=\frac{1}{2} \operatorname{tr}_{g} h \cdot d V o l_{g}$. Then we can compute the variation of $\mathcal{F}_{k}[g]$ :

$$
\begin{aligned}
\delta \mathcal{F}_{k}[\tilde{g}]= & \int_{M} \delta \sigma_{k}\left(C_{\tilde{g}}\right) d V o l_{g}+\int_{M} \sigma_{k}\left(C_{g}\right) \delta d V o l_{\tilde{g}} \\
= & \int_{M}\left\{-\sum_{i, j, l}\left(T_{k-1}\right)_{j}^{i} h^{j l} R_{i l}-\sum_{i, j, m, l}\left(T_{k-1}\right)^{i j} h^{m l} R_{i m j l}\right. \\
& -\frac{1}{2} \sum_{i, j}\left[\left(T_{k-1}\right)_{j}^{i} \Delta_{g} h_{i}^{j}+\left(T_{k-1}\right)^{i j}\left(\operatorname{tr}_{g} h\right)_{, i j}\right]+\frac{1}{2} \sigma_{k}\left(C_{g}\right) \operatorname{tr}_{g} h \\
& +\frac{1}{2} \sum_{i, j, m}\left(T_{k-1}\right)_{j}^{i}\left[h_{m i} R^{m j}+h_{m}^{j} R_{i}^{m}+\left(h^{m j}\right)_{, m i}+\sum_{l} g^{j l}\left(h_{i}^{m}\right)_{, m l}\right] \\
& \left.-\frac{1}{2(n-1)} \operatorname{tr}\left(T_{k-1}\right)\left[\sum_{i, j}\left(h^{i j}\right)_{, i j}-\sum_{i, j} h^{i j} R_{i j}-\Delta_{g}\left(\operatorname{tr}_{g} h\right)\right]\right\} d V o l_{g} .
\end{aligned}
$$

By using the Stokes' formula and (4) of Lemma 3.1, we can rewrite (3.13) as follows:

$$
\begin{aligned}
\delta \mathcal{F}_{k}[\tilde{g}]= & \int_{M} \sum_{i, j}\left\{-\frac{1}{2} \Delta_{g}\left(T_{k-1}\right)_{i j}-\sum_{m, l}\left(T_{k-1}\right)^{m l} R_{m i l j}+\sum_{l}\left(\left(T_{k-1}\right)_{i}^{l}\right)_{, l j}\right. \\
& +\frac{1}{2(n-1)} \operatorname{tr}\left(T_{k-1}\right) R_{i j}-\frac{1}{2(n-1)}\left(\operatorname{tr}\left(T_{k-1}\right)\right)_{, i j}+\frac{1}{2} \sigma_{k}\left(C_{g}\right) g_{i j} \\
& \left.-\frac{1}{2} \sum_{m, l}\left(\left(T_{k-1}\right)^{m l}\right)_{, m l} g_{i j}+\frac{1}{2(n-1)} \Delta_{g}\left(\operatorname{tr} T_{k-1}\right) g_{i j}\right\} h^{i j} d V o l_{g} .
\end{aligned}
$$


From the decomposition (2.3), (2.4) of the Riemannian curvature tensor, Lemma $3.1(1)$ and the fact $T_{k-1} C=C T_{k-1}$, we get

$$
\begin{aligned}
-\sum_{m, l}\left(T_{k-1}\right)^{m l} R_{m i l j}= & -\sum_{m, l}\left(T_{k-1}\right)^{m l} W_{m i l j}-\frac{k}{n-2} \sigma_{k}\left(C_{g}\right) g_{i j} \\
& -\frac{1}{n-2} \operatorname{tr}\left(T_{k-1}\right) C_{i j}+\frac{2}{n-2} \sum_{m}\left(T_{k-1}\right)_{i}^{m} C_{m j} .
\end{aligned}
$$

Putting (3.15) into (3.14) and then using

$$
R_{i j}=E_{i j}+\frac{r}{n} g_{i j}, \quad C_{i j}=E_{i j}+\frac{n-2}{2 n(n-1)} r g_{i j},
$$

we immediately obtain (3.1) by noting (1.1).

From Proposition 3.1, we can deduce our main result of this section.

Theorem 3.1. Suppose $M^{n}$ is compact. Then a metric $g \in \mathcal{M}_{1}$ is a critical point for $\left.\mathcal{F}_{k}\right|_{\mathcal{M}_{1}}$ if and only if it satisfies the following two equations:

$$
\begin{aligned}
& \Delta_{g}\left(T_{k-1}\right)_{i j}+2 \sum_{m, l}\left(T_{k-1}\right)^{m l} W_{i m j l}-\frac{4}{n-2} \sum_{l}\left(T_{k-1}\right)_{j}^{l} E_{i l}-2 \sum_{l}\left[\left(T_{k-1}\right)_{i}^{l}\right]_{, l} \\
& -\frac{2 r}{n(n-1)}\left(T_{k-1}\right)_{i j}+\frac{n}{(n-1)(n-2)} \operatorname{tr}\left(T_{k-1}\right) E_{i j}+\frac{4 k}{n(n-2)} \sigma_{k} g_{i j} \\
& -\frac{1}{n-1} \Delta_{g}\left(\operatorname{tr} T_{k-1}\right) g_{i j}+\frac{1}{n-1}\left(\operatorname{tr} T_{k-1}\right)_{, i j}+\frac{2}{n} \sum_{m, l}\left[\left(T_{k-1}\right)^{m l}\right]_{, m l} g_{i j}=0 .
\end{aligned}
$$

Proof. According to the principle of Lagrange's multiplier, $g \in \mathcal{M}_{1}$ is a critical point of $\left.\mathcal{F}_{k}\right|_{\mathcal{M}_{1}}$ if and only if for some constant $\lambda$, it is a critical point of the auxiliary functional

$$
\tilde{\mathcal{F}}_{k}: g \mapsto \int_{M} \sigma_{k}\left(C_{g}\right) d V o l_{g}-2 \lambda[\operatorname{Vol}(M, g)-1],
$$

defined on $\mathcal{M}$. From the proof of Proposition 3.1 we easily known that, at any fixed $g \in \mathcal{M},\left(\nabla \tilde{\mathcal{F}}_{k}\right)_{i j}=\left(\nabla \mathcal{F}_{k}\right)_{i j}-\lambda g_{i j}$. This implies that $g \in \mathcal{M}_{1}$ is a critical point of $\left.\tilde{\mathcal{F}}_{k}\right|_{\mathcal{M}}$ if and only if it satisfies

$$
\left(\nabla \mathcal{F}_{k}\right)_{i j}=\lambda g_{i j}
$$

By contracting (3.19), using $\sum_{i, j} W_{i m j l} g^{i j}=0$ and Lemma 3.1(1), we can get (3.17). Inserting (3.17) into (3.19), we obtain (3.18).

Corollary 3.1. Suppose $M^{n}$ is compact and $g \in \mathcal{M}_{1}$ is a critical point of $\left.\mathcal{F}_{k}\right|_{\mathcal{M}_{1}}$ $(n \neq 2 k)$. Then

(1) when $k=1,2$, we have $\sigma_{k}\left(C_{g}\right)=$ const on $M^{n}$;

(2) when $k \geq 3$ and $\left(M^{n}, g\right)$ is a locally conformally flat manifold, we also have $\sigma_{k}\left(C_{g}\right)=$ const on $M^{n}$. 
Proof. Since $T_{0}\left(C_{g}\right)=I, T_{1}\left(C_{g}\right)=-$ Ric $+\frac{r}{2} \mathrm{I}$, where $I$ denotes the identity transformation on $T M$, i.e., with respect to a local frames field $\left\{e_{i}\right\}$,

$$
T_{0}\left(C_{g}\right)_{i}^{j}=\delta_{i}^{j}, \quad T_{1}\left(C_{g}\right)_{i}^{j}=-\operatorname{Ric}_{i}^{j}+\frac{r}{2} \delta_{i}^{j} .
$$

Now the conclusion follows from (3.17), (4)-(5) of Lemma 3.1 and the fact that $C_{g}$ is a Codazzi tensor in case $g$ is a locally conformally flat metric.

Combining Theorem 3.1 with Lemma 3.1(4) and the proof of Corollary 3.1, we immediately have

Corollary 3.2. Suppose $M^{n}$ is compact and $g \in \mathcal{M}_{1}$ is a locally conformally flat metric. Then $g$ is a critical point of $\mathcal{F}_{k} \mid \mathcal{M}_{1}(n \neq 2 k)$ if and only if it satisfies the conditions that $\sigma_{k}\left(C_{g}\right)=$ const and

$$
\begin{aligned}
& \Delta_{g}\left(T_{k-1}\right)_{i j}-\frac{1}{n-1} \Delta_{g}\left(\operatorname{tr} T_{k-1}\right) g_{i j}+\frac{1}{n-1}\left(\operatorname{tr} T_{k-1}\right)_{, i j}+\frac{4 k}{n(n-2)} \sigma_{k} g_{i j} \\
& -\frac{4}{n-2} \sum_{l}\left(T_{k-1}\right)_{j}^{l} E_{i l}-\frac{2 r}{n(n-1)}\left(T_{k-1}\right)_{i j}+\frac{n}{(n-1)(n-2)} \operatorname{tr}\left(T_{k-1}\right) E_{i j}=0 .
\end{aligned}
$$

Remark 3.2. The condition $n \neq 2 k$ is natural due to the fact that, as has been observed by Viaclovsky in [10, if $n=2 k$ and $M$ carries a locally conformally flat metric, then $\mathcal{F}_{k}[g]$ is an invariant and, in fact, is a multiple of the Euler characteristic of $M$ for locally conformally flat metric $g$.

\section{General properties for critical points of $\left.\mathcal{F}_{2}\right|_{\mathcal{M}_{1}}$}

From now on, we restrict our attention to $\left.\mathcal{F}_{2}\right|_{\mathcal{M}_{1}}$ with $n \neq 4$. On the one hand, we have

Proposition 4.1. Suppose $M^{n}(n \neq 4)$ is compact, and a metric $g \in \mathcal{M}_{1}$ is a critical point of $\mathcal{F}_{2} \mid \mathcal{M}_{1}$. Then, with respect to a local orthonormal frame field $\left\{e_{i}\right\}$ of $g$, the norm square of the trace-free Ricci tensor $E=R i c_{g}-\frac{r}{n} g$ satisfies

$$
\begin{aligned}
\frac{1}{2} \Delta_{g} \sum_{i, j}\left(E_{i j}\right)^{2}= & \sum_{i, j, k}\left(E_{i j, k}\right)^{2}+\frac{n-2}{2(n-1)} \sum_{i, j} E_{i j} r_{, i j}+\frac{4}{n-2} \sum_{i, j, k} E_{i j} E_{j k} E_{k i} \\
& +\frac{n^{2}-4 n+8}{2 n(n-1)} r \sum_{i, j}\left(E_{i j}\right)^{2}-2 \sum_{i, j, m, l} E_{i j} E_{m l} W_{m i l j} .
\end{aligned}
$$

Proof. Using the identities (3.16) and (3.20), we have

$$
\left(T_{1}\left(C_{g}\right)\right)_{i j}=-E_{i j}+\frac{n-2}{2 n} r \delta_{i j} .
$$

By use of (4.2) and the fact that $T_{1}$ has vanishing divergence, we have from

$$
\begin{aligned}
\Delta_{g} E_{i j}= & \frac{n-2}{2(n-1)} r_{, i j}-\frac{n-2}{2 n(n-1)} \Delta_{g} r \delta_{i j}-2 \sum_{m, l} E_{m l} W_{i m j l}-\frac{n-2}{n^{2}(n-1)} r^{2} \delta_{i j} \\
& +\frac{4}{n-2} \sum_{l} E_{i l} E_{l j}+\frac{n^{2}-4 n+8}{2 n(n-1)} r E_{i j}+\frac{8}{n(n-2)} \sigma_{2} \delta_{i j} .
\end{aligned}
$$


Combining (4.3) with

$$
\frac{1}{2} \Delta_{g} \sum_{i, j}\left(E_{i j}\right)^{2}=\sum_{i, j, k}\left(E_{i j, k}\right)^{2}+\sum_{i, j} E_{i j} \Delta_{g} E_{i j},
$$

we get (4.1).

On the other hand, we can prove

Proposition 4.2. Let $\left(M^{n}, g\right)$ be a locally conformally flat manifold. Then, with respect to an orthonormal frame field $\left\{e_{i}\right\}$ of $g$, we have

$$
\begin{aligned}
& \frac{1}{2} \Delta_{g} \sum_{i, j}\left(E_{i j}\right)^{2} \\
& =\sum_{i, j, k}\left(E_{i j, k}\right)^{2}+\frac{n-2}{2(n-1)} \sum_{i, j} E_{i j} r_{, i j}+\frac{n}{n-2} \sum_{i, j, l} E_{i l} E_{l j} E_{j i}+\frac{r}{n-1} \sum_{i, j}\left(E_{i j}\right)^{2} .
\end{aligned}
$$

Proof. By use of (3.16) and (2.6)-(2.8), we have

$$
\begin{aligned}
\Delta_{g} E_{i j} & =\Delta_{g} C_{i j}-\frac{n-2}{2 n(n-1)} \Delta r \cdot g_{i j} \\
& =\sum_{l} C_{l l, i j}+\sum_{m, l}\left(C_{m i} R_{m l j l}+C_{m l} R_{m i j l}\right)-\frac{n-2}{2 n(n-1)} \Delta r \cdot g_{i j} \\
& =\frac{n-2}{2(n-1)} r_{, i j}-\frac{n-2}{2 n(n-1)} \Delta_{g} r \delta_{i j} \\
& +\frac{n}{n-2} \sum_{l} E_{i l} E_{l j}-\frac{1}{n-2} \sum_{m, l}\left(E_{m l}\right)^{2} \delta_{i j}+\frac{r}{n-1} E_{i j} .
\end{aligned}
$$

Putting (4.6) into (4.4), we get (4.5).

From (3.16), a simple calculation gives

$$
\sigma_{2}\left(C_{g}\right)=-\frac{1}{2}|E|^{2}+\frac{(n-2)^{2}}{8 n(n-1)} r^{2} .
$$

Comparing (4.3) with (4.6), and then making use of (4.7) and Corollary 3.2 in the case $k=2$, we immediately obtain the following

Corollary 4.1. Suppose $M^{n}(n \neq 4)$ is compact. Then a locally conformally flat metric $g \in \mathcal{M}_{1}$ is a critical point of $\left.\mathcal{F}_{2}\right|_{\mathcal{M}_{1}}$ if and only if $\sigma_{2}\left(C_{g}\right)=$ const and the following algebraic identities hold:

$$
\begin{gathered}
\sum_{l} E_{i}^{l} E_{l j}-\frac{(n-2)^{2}}{2 n(n-1)} r E_{i j}-\frac{1}{n}|E|^{2} g_{i j}=0, \\
\operatorname{tr}_{g} E^{3}-\frac{(n-2)^{2}}{2 n(n-1)} r|E|^{2}=0 .
\end{gathered}
$$




\section{LOCALLY CONFORMALLY FLAT CRITICAL $g$ OF $\left.\mathcal{F}_{2}\right|_{\mathcal{M}_{1}}$ With $\mathcal{F}_{2}[g]>0$}

We first state an algebraic lemma.

Lemma 5.1 (see [7] or [5]). For any real numbers $a_{1}, \cdots, a_{n}$ with $\sum_{i} a_{i}=0$, there holds

$$
-\frac{n-2}{\sqrt{n(n-1)}}\left(\sum_{i=1}^{n} a_{i}^{2}\right)^{3 / 2} \leq \sum_{i=1}^{n} a_{i}^{3} \leq \frac{n-2}{\sqrt{n(n-1)}}\left(\sum_{i=1}^{n} a_{i}^{2}\right)^{3 / 2},
$$

and equality holds in (5.1) if and only if at least $n-1$ of the $a_{i}$ 's are equal. In particular, for $\sum_{i=1}^{n} a_{i}^{2} \neq 0$, if $\sum_{i=1}^{n} a_{i}^{3}=-\frac{n-2}{\sqrt{n(n-1)}}\left(\sum_{i=1}^{n} a_{i}^{2}\right)^{3 / 2}$, then the $n-1$ of the $a_{i}$ 's which are equal must be positive; if $\sum_{i=1}^{n} a_{i}^{3}=\frac{n-2}{\sqrt{n(n-1)}}\left(\sum_{i=1}^{n} a_{i}^{2}\right)^{3 / 2}$, then the $n-1$ of the $a_{i}$ 's which are equal must be negative.

Now, we suppose that $\left(M^{n}, g\right)$ is a compact locally conformally flat manifold and $g \in \mathcal{M}_{1}$ is a critical point of $\left.\mathcal{F}_{2}\right|_{\mathcal{M}_{1}}$. Then, according to Corollary $3.2, \sigma_{2}\left(C_{g}\right)=$ const. To prove Theorem B, we first consider the case $\mathcal{F}_{2}[g]>0$, i.e., $\sigma_{2}\left(C_{g}\right)=$ const $>0$.

Proposition 5.1. Suppose $M^{n}(n \neq 4)$ is compact, and $g \in \mathcal{M}_{1}$ is a locally conformally flat metric. If $g$ is a critical point of $\left.\mathcal{F}_{2}\right|_{\mathcal{M}_{1}}$ with $\sigma_{2}\left(C_{g}\right)>0$, then $\left(M^{n}, g\right)$ is a space form.

Proof. To prove the proposition, it suffices to show that $|E| \equiv 0$ on $M^{n}$. If it is not the case, then there exists a point $p \in M^{n}$ such that $|E|(p) \neq 0$. We will derive a contradiction.

Note that $\sigma_{2}\left(C_{g}\right)>0$ and (4.7) imply that

$$
\frac{n-2}{2 \sqrt{n(n-1)}}|r|>|E| \geq 0, \text { on } M^{n} .
$$

Since $M^{n}$ is connected, from (5.2) we have only two possible cases: $r>0$ on $M^{n}$ or $r<0$ on $M^{n}$.

If $r>0$, then at $p$, by applying Lemma 5.1, we have from (4.9) and (5.2)

$$
\begin{aligned}
0 & =\operatorname{tr}_{g} E^{3}-\frac{(n-2)^{2}}{2 n(n-1)} r|E|^{2} \leq \frac{n-2}{\sqrt{n(n-1)}}|E|^{3}-\frac{(n-2)^{2}}{2 n(n-1)} r|E|^{2} \\
& <\frac{n-2}{\sqrt{n(n-1)}}|E|^{3}-\frac{n-2}{\sqrt{n(n-1)}}|E|^{3}=0
\end{aligned}
$$

which is a contradiction.

If $r<0$, then at $p$, from (4.9) and (5.2) and applying Lemma 5.1, we have

$$
\begin{aligned}
0 & =\operatorname{tr}_{g} E^{3}-\frac{(n-2)^{2}}{2 n(n-1)} r|E|^{2} \geq-\frac{n-2}{\sqrt{n(n-1)}}|E|^{3}-\frac{(n-2)^{2}}{2 n(n-1)} r|E|^{2} \\
& >-\frac{n-2}{\sqrt{n(n-1)}}|E|^{3}+\frac{n-2}{\sqrt{n(n-1)}}|E|^{3}=0,
\end{aligned}
$$

which is also a contradiction. This completes the proof of Proposition 5.1. 


\section{Locally CONFORMally flat CRitical Metric $g$ OF $\left.\mathcal{F}_{2}\right|_{\mathcal{M}_{1}}$ With $\mathcal{F}_{2}[g]=0$}

In this section, we find that the analysis of [4] can be carried through by some modifications so as to extend the 3 -dimensional results there to any dimension $n \geq 5$ for conformally flat critical points of $\left.\mathcal{F}_{2}\right|_{\mathcal{M}_{1}}$.

We first recall a result about the elementary symmetric function $S_{2}\left(x_{1}, \cdots, x_{n}\right)$ defined by (2.10). Note that as a homogeneous function of degree two, $S_{2}$ has signature $(1, n-1)$ with index $n-1$. Thus the set $\left\{x \in R^{n} \mid S_{2}(x)>0\right\}$ has exactly two components. Following [4], we let $\Gamma_{2}^{+}$denote the component which contains the positive cone. For a symmetric linear transformation $C: V^{n} \rightarrow V^{n}$, where $V^{n}$ is an $n$-dimensional inner product space, the notation $C \in \Gamma_{2}^{+}$will mean that the eigenvalues $\left(\lambda_{1}, \cdots, \lambda_{n}\right)$ of $C$ lies in $\Gamma_{2}^{+}$. We will need the following important properties of $\Gamma_{2}^{+}$.

Lemma 6.1 (cf. [4]). (1) The set $\Gamma_{2}^{+}$is an open convex cone with vertex at the origin.

(2) If $C \in \Gamma_{2}^{+}$, then the Newtonian $T_{1}(C)$ defined by (2.12) is positive definite.

(3) $\Gamma_{2}^{+} \subset \Gamma_{1}^{+}:=\left\{x \in R^{n} \mid S_{1}(x)>0\right\}$.

Now we present a global characterization for the null criticals, i.e., metrics $g$ which are critical for $\left.\mathcal{F}_{2}\right|_{\mathcal{M}_{1}}$ with $\mathcal{F}_{2}[g]=0$.

Theorem 6.1. Let $\left(M^{n}, g\right)(n \geq 5)$ be compact and let $g \in \mathcal{M}_{1}$ be a conformally flat metric. Then $g$ is a critical point of $\left.\mathcal{F}_{2}\right|_{\mathcal{M}_{1}}$ with $\mathcal{F}_{2}[g]=0$ if and only if $r \leq 0$ and the eigenvalues of the tensor $C_{g}$ are $\{\underbrace{0, \cdots, 0}_{n-1}, \frac{n-2}{2(n-1)} r\}$.

Proof. The if part is a direct check with application of Corollary 4.1. We will only give a detailed proof for the only if part. We first prove our claim that $r \leq 0$.

Note that $\mathcal{F}_{2}[g]=0$ implies $\sigma_{2}\left(C_{g}\right)=0$ and

$$
|E|^{2}=\frac{(n-2)^{2}}{4 n(n-1)} r^{2} .
$$

Then by (4.2) and (4.3), we easily get

$$
0=|\nabla E|^{2}-\frac{n-2}{2(n-1)} \sum_{i, j}\left(T_{1}\left(C_{g}\right)\right)_{i j} r_{, i j}+\frac{n}{n-2} \operatorname{tr} E^{3}+\frac{r}{n-1}|E|^{2}-\frac{(n-2)^{2}}{4 n(n-1)}|\nabla r|^{2} .
$$

Let $p \in M^{n}$ be a point where $r$ achieves its maximum. Then $\nabla r(p)=0$ and $\left(r_{, i j}(p)\right)$ is negative semi-definite. If $r(p) \geq 0$, then

$$
\sigma_{1}\left(C_{g}\right)(p)=\left(\operatorname{tr}_{g} C_{g}\right)(p)=\frac{n-2}{2(n-1)} r(p) \geq 0 .
$$

Because $\sigma_{2}\left(C_{g}\right)(p)=0$, from Lemma 6.1 we conclude that $p$ must be on the boundary of the positive cone $\Gamma_{2}^{+}$and $T_{1}\left(C_{g}\right)(p)$ is positive semi-definite. Now (6.2) implies

$$
0 \geq|\nabla E|^{2}(p)+\frac{n}{n-2} \operatorname{tr} E^{3}(p)+\frac{r(p)}{n-1}|E|^{2}(p)=|\nabla E|^{2}(p)+\frac{n}{2(n-1)} r(p)|E|^{2}(p),
$$

where we used (4.9) in the last step. Therefore $r(p) \cdot|E|(p)=0$ which implies $r(p)=0$ by (6.1), so $r \leq 0$ everywhere on $M^{n}$. 
Now from (4.9), (6.1) and the fact that $r \leq 0$ on $M^{n}$, we get

$$
\operatorname{tr}_{g} E^{3}=-\frac{n-2}{\sqrt{n(n-1)}}|E|^{3} .
$$

By Lemma 5.1 we see that the eigenvalues of $E$ are of the form $\{a, \cdots, a,-(n-1) a\}$ for some function $a \geq 0$. Then we deduce from (3.16) that the eigenvalues $\left\{\lambda_{i}\right\}$ of $C_{g}$ satisfy

$$
\lambda_{1}=\cdots=\lambda_{n-1}=a+\frac{n-2}{2 n(n-1)} r, \lambda_{n}=-(n-1) a+\frac{n-2}{2 n(n-1)} r .
$$

Now we have $|E|^{2}=n(n-1) a^{2}$, which in combination with (6.1) gives $a=$ $-\frac{n-2}{2 n(n-1)} r$. Therefore we find $\lambda_{1}=\cdots=\lambda_{n-1}=0$ and $\lambda_{n}=\frac{n-2}{2(n-1)} r$. This proves Theorem 6.1.

Theorem 6.2. Let $M^{n}(n \geq 5)$ be compact and let $g \in \mathcal{M}_{1}$ be a conformally flat metric. If $g$ is critical for $\left.\mathcal{F}_{2}\right|_{\mathcal{M}_{1}}$ with $\mathcal{F}_{2}[g]=0$, then for each $p \in M^{n}$, either

(i) the sectional curvature vanishs at $p$, or

(ii) there exists a local coordinate system $\left\{x_{1}, \cdots, x_{n-1}, y\right\}$ around $p$ mapping a neighborhood of $p$ to a cube in $R^{n}$ in which the metric $g$ takes the form

$$
g=d x_{1}^{2}+\cdots+d x_{n-1}^{2}+f\left(x_{1}, \cdots, x_{n-1}, y\right)^{2} d y^{2}
$$

with

$$
f\left(x_{1}, \cdots, x_{n-1}, y\right)=a(y) \sum_{i=1}^{n-1} x_{i}^{2}+\sum_{i=1}^{n-1} b_{i}(y) x_{i}+c(y),
$$

where $a(y),\left\{b_{i}(y)\right\}, c(y)$ are some functions of $y$.

Proof. If $r=0$ at $p$, then (i) holds. We may therefore assume, by Theorem 6.1, that $r<0$ around $p$. Then in a neighborhood of $p, r \neq 0$ and $T M$ has a decomposition as $T M=V_{1} \oplus V_{2}$, where $V_{1}$ and $V_{2}$ are the eigenspaces of the tensor $C_{g}$ with eigenvalues 0 and $\frac{n-2}{2(n-1)} r$, where $\operatorname{dim} V_{1}=n-1, \operatorname{dim} V_{2}=1$.

Since $C_{g}$ is a Codazzi tensor, according to A. Derziński [3], $V_{1}$ is an integrable distribution. $V_{2}$ is a 1-dimensional distribution, therefore it is also integrable. Thus we have a local coordinate system $\left\{x_{1}, \cdots, x_{n}\right\}$ mapping a neighborhood of $p \in M^{n}$ to a cube in $R^{n}$ with $\operatorname{Span}\left\{\frac{\partial}{\partial x_{1}}, \cdots, \frac{\partial}{\partial x_{n-1}}\right\}=V_{1}$ and $\operatorname{Span}\left\{\frac{\partial}{\partial x_{n}}\right\}=V_{2}$.

Since eigenspaces corresponding to distinct eigenvalues are orthogonal, we have that the metric $g$ locally takes the form

$$
\left(\begin{array}{cc}
\left(g_{i j}(x)\right)_{(n-1) \times(n-1)} & 0 \\
0 & g_{n n}(x)
\end{array}\right) .
$$

Claim 6.1. Integral manifolds of $V_{1}$ are flat and totally geodesic submanifolds of $M^{n}$.

Proof. Since $V_{1}$ is the eigenspace of a Codazzi tensor with the constant eigenvalue 0 , by [3], the integral manifolds of $V_{1}$ are totally geodesic submanifolds of $M^{n}$. Let $\left\{e_{i}\right\}_{1 \leq i \leq n-1}$ be an orthonormal frame field on $V_{1}$. Since $\left(M^{n}, g\right)$ is conformally flat, from $(2.3)$ we have

$$
R\left(e_{i}, e_{j}, e_{i}, e_{j}\right)=\frac{1}{n-2}\left(C_{i i}+C_{j j}\right)=0, \quad \forall i \neq j .
$$


Therefore, from the Gauss equation, the integral manifolds of $V_{1}$ are of zero sectional curvature and thus are flat.

Let us consider a slice of an integral manifold $N:=\left\{x_{n}=\right.$ const $\}$ of $V_{1}$. Let $\Pi$ denote the second fundamental form of $N \hookrightarrow M^{n}$. Note that $\left\{x_{1}, \cdots, x_{n-1}\right\}$ forms a local coordinate system on $N$. Denote $\partial_{a}=\frac{\partial}{\partial x_{a}}$. In the sequel we will make use of the following convention on the range of indices: $1 \leq i, j, k, l \leq n-1 ; 1 \leq$ $a, b, c, d \leq n$.

Since $e_{n}=g_{n n}^{-1 / 2} \partial_{n}$ is the unit normal vector of $N \hookrightarrow M^{n}$, from Claim 6.1 we have

$$
0=\Pi\left(\partial_{i}, \partial_{j}\right)=-g\left(\nabla_{\partial_{i}} e_{n}, \partial_{j}\right)=-g_{n n}^{-1 / 2} \sum_{a} \Gamma_{n i}^{a} g_{j a}=-\frac{1}{2} g_{n n}^{-1 / 2} \partial_{n} g_{i j},
$$

i.e., $\partial_{n} g_{i j}=0,1 \leq i, j \leq n-1$. Therefore, since the slice $N$ is flat, by changing the coordinates $\left\{x_{1}, \cdots, x_{n-1}\right\}$ if necessary, we can assume that the metric has the local form: $g_{i j}=\delta_{i j}$ and $g_{n a}=f^{2}\left(x_{1}, \cdots, x_{n}\right) \delta_{n a}$ for some positive function $f(x)>0$.

\section{Claim 6.2.}

$$
\frac{\partial^{2} f}{\partial x_{i} \partial x_{j}}=\frac{\partial^{2} f}{\partial x_{1}^{2}} \delta_{i j}, \quad 1 \leq i, j \leq n-1 .
$$

Proof. Since $\partial_{n} g_{i j}=0=g_{n i}, \forall i, j$, by a simple calculation we have

$$
\left\{\begin{array}{l}
\Gamma_{n n}^{i}=-\frac{1}{2} \partial_{i} g_{n n}=-f \partial_{i} f \\
\Gamma_{n i}^{n}=\Gamma_{i n}^{n}=\frac{1}{2} g^{n n} \partial_{i} g_{n n}=\partial_{i} \log f, 1 \leq i \leq n-1, \\
\Gamma_{n n}^{n}=\frac{1}{2} g^{n n} \partial_{n} g_{n n}=\partial_{n} \log f \\
\Gamma_{a b}^{c}=0, \text { for all other cases. }
\end{array}\right.
$$

According to (3.5), the components of the Ricci tensor are given by

$$
R_{a b}=\sum_{c} R_{a c b}^{c}=\sum_{c}\left(\partial_{c} \Gamma_{a b}^{c}-\partial_{b} \Gamma_{a c}^{c}\right)+\sum_{c, d}\left(\Gamma_{c d}^{c} \Gamma_{a b}^{d}-\Gamma_{b d}^{c} \Gamma_{a c}^{d}\right) .
$$

From (6.5) and (6.6), we have

$$
R_{i j}=-f^{-1} \frac{\partial^{2} f}{\partial x_{i} x_{j}}, \quad R_{n i}=0, \quad 1 \leq i, j \leq n-1 ; \quad R_{n n}=-\sum_{i} f \frac{\partial^{2} f}{\partial x_{i}^{2}} .
$$

Then we get the scalar curvature as follows:

$$
r=\sum_{a, b} g^{a b} R_{a b}=\sum_{i} R_{i i}+f^{-2} R_{n n}=-2 f^{-1} \sum_{i} \frac{\partial^{2} f}{\partial x_{i}^{2}} .
$$

From (2.4) and the above calculation, we have

$$
\left\{\begin{array}{l}
C_{i j}=R_{i j}=-f^{-1} f_{i j}, \quad i \neq j, \\
C_{i i}=R_{i i}-\frac{r}{2(n-1)}=-f^{-1} f_{i i}+\frac{1}{(n-1) f} \sum_{j} f_{j j}, \\
C_{n n}=R_{n n}-\frac{r}{2(n-1)}=\frac{2-n}{n-1} f \sum_{i} f_{i i}, \quad C_{n i}=0,
\end{array}\right.
$$

where we denote $f_{i j}=\frac{\partial^{2} f}{\partial x_{i} \partial x_{j}}$.

Since all the eigenvalues of $\left(C_{i j}\right)_{(n-1) \times(n-1)}$ are zero, we have $C_{i j} \equiv 0, \quad 1 \leq$ $i, j \leq n-1$. From this and (6.7) we conclude that $f_{i j}=0$ when $i \neq j$. Note that $C_{11}=\cdots=C_{n-1, n-1}=0$ and (6.7) imply $R_{11}=\cdots=R_{n-1, n-1}$, which gives 
$f_{11}=\cdots=f_{n-1, n-1}$. Then from (6.7) again we find $C_{n n}=-(n-2) f f_{i i}$ for any $1 \leq i \leq n-1$. This proves our Claim 6.2.

Claim 6.3. $f_{i i i}=0, \quad 1 \leq i \leq n-1$.

Proof. From the fact that $C_{g}$ is a Codazzi tensor, we have

$$
\nabla_{i} C_{n n}=\nabla_{n} C_{n i}, 1 \leq i \leq n-1 .
$$

From the direct calculation

$$
\begin{aligned}
& \nabla_{i} C_{n n}=\partial_{i}\left(C_{n n}\right)-2 \sum_{a} \Gamma_{n i}^{a} C_{n a}=(n-2) f_{i} f_{i i}-(n-2) f f_{i i i}, \\
& \nabla_{n} C_{n i}=\partial_{n}\left(C_{n i}\right)-\sum_{a} \Gamma_{n n}^{a} C_{a i}-\sum_{a} \Gamma_{n i}^{a} C_{n a}=-\sum_{a} \Gamma_{n i}^{n} C_{n n}=(n-2) f_{i} f_{i i},
\end{aligned}
$$

we find that $f f_{i i i}=0,1 \leq i \leq n-1$. Then Claim 6.3 follows.

From Claims 6.2 and 6.3, we have finished the proof of Theorem 6.2.

Proposition 6.1. Let $g$ be a metric on $R^{n}=\left\{x_{1}, \cdots, x_{n-1}, x_{n}\right\}$ of the form

$$
g=d x_{1}^{2}+\cdots+d x_{n-1}^{2}+f\left(x_{1}, \cdots, x_{n-1}, x_{n}\right)^{2} d x_{n}^{2}
$$

with

$$
f\left(x_{1}, \cdots, x_{n-1}, x_{n}\right)=a\left(x_{n}\right) \sum_{i=1}^{n-1} x_{i}^{2}+\sum_{i=1}^{n-1} b_{i}\left(x_{n}\right) x_{i}+c\left(x_{n}\right) .
$$

Then $g$ is locally conformally flat and satisfies $\sigma_{2}\left(C_{g}\right)=0$. Furthermore, $g$ is critical for $\mathcal{F}_{2}$ with respect to all compactly supported variations.

Proof. Simple calculations show that the metric $g$ satisfies (6.5) and the following relations:

(i) $R_{i j}=-f^{-1} f_{i j}$; so $R_{11}=\cdots=R_{n-1, n-1}, R_{i j}=0, i \neq j ; 1 \leq i, j \leq n-1$.

$$
R_{n i}=0, \quad 1 \leq i \leq n-1 ; \quad R_{n n}=-(n-1) f f_{11} ; \quad r=-2(n-1) f^{-1} f_{11} .
$$

(ii) $C_{i j}=0 ; \quad C_{n i}=0 ; \quad C_{n n}=-(n-2) f f_{11}, \quad 1 \leq i, j \leq n-1$.

(iii) $C_{g}$ is a Codazzi tensor.

By formula (3.5) of the full curvature tensor $R_{a b c d}$, a direct check proves that $C$ and $g$ satisfy $R_{a b c d}=\frac{1}{n-2}\left(C_{a c} g_{b d}-C_{a d} g_{b c}+C_{b d} g_{a c}-C_{b c} g_{a d}\right)$. This and (iii) verify that $\left(R^{n}, g\right)$ is a non-compact locally conformally flat Riemannian manifold.

From (ii) above, we see that $\sigma_{2}\left(C_{g}\right)=0$. Now we can also check that (4.8) is satisfied. Then, by making use of (4.6) and (4.8), we obtain from (3.1) that $\nabla \mathcal{F}_{2}=0$, which implies that $g$ is critical for $\mathcal{F}_{2}$ with respect to all compactly supported variations. This proves Proposition 6.1.

Remark 6.1. Similar to the 3-dimensional situation in 4], Proposition 6.1 implies that, for higher dimension $n \geq 4$, there might exist abundance complete null critical metrics of non-constant sectional curvature on a non-compact manifold, e.g. $R^{n}$. Here we notice that, by appropriately choosing the functions $\left\{a\left(x_{n}\right), b_{i}\left(x_{n}\right), c\left(x_{n}\right)\right\}$ in (6.8), the Riemannian manifold $\left(R^{n}, g\right)$ is complete, e.g., $f\left(x_{1}, \cdots, x_{n}\right)=1+$ $x_{1}^{2}+\cdots+x_{n}^{2}$.

To prove Theorem B for the null critical case, it suffices to show the scalar curvature $r \equiv 0$ on $M^{n}$. Since we have proved in Theorem 6.1 that $r \leq 0$ on $M^{n}$, we now consider the set $M_{-} \equiv\{p \in M: r(p)<0\}$. If $M_{-}=\varnothing$, then we are done. 
So in the sequel we assume $M_{-} \neq \varnothing$. To derive a needed contradiction, we will adopt the method due to Gursky and Viaclovsky (cf. 4]) by undertaking a careful study of the leaves of the foliation of $M_{-}$defined by the distribution $V_{1}$ in Theorem 6.2. Notice that $V_{1}$ is integrable, so by the well-known Frobenius Integrability Theorem, we are guaranteed the existence of a unique maximal connected integral manifold through each point where the scalar curvature is negative.

We first present a result which is crucial for our proof of Theorem B in the null critical case.

Proposition 6.2. Let $i: N \hookrightarrow M_{-}$be a maximal connected integral manifold of $V_{1}$. Then $N$ is isometric to $R^{n-1}$ with the flat metric, and $i$ is a proper imbedding.

Proof. By definition, $i$ is an injective immersion. From Claim 6.1, we know that $N$ is a flat $(n-1)$-dimensional Riemannian manifold and $i$ is a totally geodesic isometric immersion.

To prove the completeness of $N$, we first prove the following

Lemma 6.2. Define a function $S$ on $N$ by $S(p)=[r(i(p))]^{-1}, \forall p \in N$. Then the Hessian of $S$ is given by

$$
D_{N}^{2}(S)=-\frac{1}{2(n-1)} g_{N}
$$

Proof. We will use the notations and conclusions in Theorem 6.2. Given $p \in N$, we can find a local coordinate system $\left\{x_{1}, \cdots, x_{n-1}, x_{n}\right\}$ around $i(p)$ such that the immersion $i$ is modeled by $\left(x_{1}, \cdots, x_{n-1}\right) \mapsto\left(x_{1}, \cdots, x_{n-1}, 0\right)$ and the components of the metric $g$ is given by $g_{i j}=\delta_{i j}, g_{n i}=0, g_{n n}=f^{2}, 1 \leq i, j \leq n-1$. From the proof of Theorem 6.2, $r=-2(n-1) f^{-1} f_{11}$ and $f_{11}=\cdots=f_{n-1, n-1}$, so we have

$$
S=-\frac{f}{2(n-1) f_{11}} \text {. }
$$

From Claim 6.3 we have $\left.f_{11}\right|_{N}=$ const. The flatness of $N$ implies that $D_{N}^{2}(S)\left(\partial_{i}, \partial_{j}\right)=\partial_{i} \partial_{j} S$. Then from Claim 6.2 , we have

$D_{N}^{2}(S)\left(\partial_{i}, \partial_{j}\right)=\partial_{i} \partial_{j}\left(-\frac{f}{2(n-1) f_{11}}\right)=-\frac{f_{i j}}{2(n-1) f_{11}}=-\frac{1}{2(n-1)} \delta_{i j}=-\frac{1}{2(n-1)} g_{i j}$.

This proves Lemma 6.2.

The following proof of Proposition 6.2 is almost identical with that in [4] except that in the second step we make use of Cheeger-Gromoll's splitting theorem. For the reader's convenience, we will keep it here.

We will now use Lemma 6.2 to show that $N$ is necessarily complete. Let $\gamma$ : $(a, b) \rightarrow N$ be any bounded geodesic segment in $N$. To show that $N$ is complete, it is sufficient to show that we can extend $\gamma$ to a longer segment in $N$. Since $i$ is a totally geodesic immersion, $i \circ \gamma$ is a geodesic segment in $M_{-}$. From (6.9), $S$ restricted to $\gamma$ is a quadratic function of the arc length, so $S$ is bounded on $\gamma$. Therefore $r \leq-c<0$ on the image of $i \circ \gamma$ and at the endpoints of $\gamma, r$ is negative and thus the endpoints of $i \circ \gamma$ are in the interior of the open set $M_{-} \subset M$. Thus we may extend $i \circ \gamma$ to a longer geodesic segment in $M_{-}$. Applying Theorem 6.2 at the endpoints of $i \circ \gamma$, we see that $N$ can be extended so that its image strictly contains the extension of $i \circ \gamma$. Since $N$ is a maximal leaf, this proves that $N$ is complete. 
Next we show that $N$ is necessarily isometric to $R^{n-1}$. From (6.9), we see that $S$ is a globally concave function on $N$. If $N$ were compact, then $S$ would attain a minimum and the Hessian would be positive semidefinite at that point, a contradiction to (6.9). Therefore $N$ is in fact a complete, non-compact and flat $(n-1)$-manifold. Then, by Cheeger and Gromoll's Splitting Theorem (cf. [2]), $N$ must be either isometric to $R^{n-1}$ or isometric to $R^{l} \times \bar{N}^{n-l-1}$ for some manifold $\bar{N}$ which contains no geodesic lines. In our case, if $\operatorname{dim} \bar{N} \geq 2$, then $\bar{N}$ is a flat manifold. Therefore in the latter case, $\bar{N}$ cannot be simply connected, which implies that there exists a closed geodesic on $N$. By restricting $S$ to such a closed geodesic, it would attain a minimum, contradicting (6.9). Therefore $N$ is isometric to $R^{n-1}$, as claimed.

Finally we show that $i: N \rightarrow M_{-}$is a proper imbedding. Let $K$ be a compact subset of $M_{-}$. Then $r \leq-c<0$ on $K$. Since $N$ was shown to be $R^{n-1}$, the local coordinate system $\left\{x_{i}\right\}_{1 \leq i \leq n-1}$ becomes a global one. Then (6.9) shows that $S$ is a function of a strictly concave quadratic polynomial in $\left\{x_{i}\right\}$. Therefore $r \circ i=S^{-1} \rightarrow$ 0 as $\sum_{i=1}^{n-1} x_{i}^{2} \rightarrow \infty$. Now $r \leq-c<0$ on $K$ implies that $i^{-1}(K)$ lies in a compact set and this proves that $i$ is proper. Because the maximal integral manifold passing through a fixed point is unique, we see that $i$ is in fact an imbedding. We have completed the proof of Proposition 6.2.

\section{Completion of the proof of Theorem B}

Because of Corollary 3.1, Proposition 5.1, Theorem 6.1 and Theorem 6.2, to prove Theorem $\mathrm{B}$ it is now sufficient to derive a contradiction in the case $M_{-} \neq \varnothing$ for the null critical case.

For any leaf $i: N \rightarrow M_{-}$of $V_{1}$, we have that $i \circ \exp _{N}=\exp _{M} \circ i_{*}$ since $i$ is totally geodesic. Therefore for any $p \in M_{-}$, $\exp _{M}$ restricted to $V_{1}(p)$ is a maximal connected integral manifold through $p$ and, by Proposition 6.2 , is a properly imbedded $R^{n-1}$. We now fix $p \in M_{-}$and let $\beta:(-\varepsilon, \varepsilon) \rightarrow M_{-}$be an integral curve of $V_{2}$ (in Theorem 6.2) passing through $p$. We identify $V_{1}(\beta(t))$ with $R^{n-1}$, and consider the normal exponential map $\Phi_{t}$ along $\beta$. Define

$$
\Phi: R^{n-1} \times(-\varepsilon, \varepsilon) \rightarrow M_{-}
$$

by $\Phi\left(x_{1}, \cdots, x_{n-1}, t\right)=\Phi_{t}\left(x_{1}, \cdots, x_{n-1}\right): R^{n-1} \rightarrow M_{-}$. As observed above, for each $t$, the map $\Phi_{t}: R^{n-1} \rightarrow M_{-}$gives a maximal integral manifold of $V_{1}$ and by Proposition $6.2, \Phi_{t}$ is further a proper imbedding. Now, by completely the same argument as in the 3 -dimensional case of [4], p. 272, we can show that for $\varepsilon$ small enough, the map $\Phi$ is an imbedding.

Claim 7.1. For $\Phi: R^{n-1} \times(-\varepsilon, \varepsilon) \rightarrow\left(M_{-}, g\right)$, we have

$$
\Phi^{*} g=d x_{1}^{2}+\cdots+d x_{n-1}^{2}+f\left(x_{1}, \cdots, x_{n-1}, t\right)^{2} d t^{2}
$$

where

$$
f\left(x_{1}, \cdots, x_{n-1}, t\right)=a(t) \sum_{i=1}^{n-1} x_{i}^{2}+\sum_{i=1}^{n-1} b_{i}(t) x_{i}+c(t)
$$

with $a(t)>0, c(t)>0$.

Proof. The form (7.2) and (7.3) just follow from the proof of Theorem 6.2. Since we are writing the metric in the form (7.2), without loss of generality we may 
assume $f>0$. Then for small $t$, we must have $c(t)>0$. From the proof of Claim 6.2, we have that $r\left(x_{1}, \cdots, x_{n-1}, t\right)=-4(n-1) a(t) f^{-1}\left(x_{1}, \cdots, x_{n-1}, t\right)$ and $r(0, \cdots, 0, t)=-4(n-1) a(t) / c(t)$. Therefore for small $t$, we must have $a(t)>0$.

Now we consider the image $U=\Phi\left(R^{n-1} \times(-\varepsilon, \varepsilon)\right)$ which is an open subset of $M$. Since the map $\Phi$ is an imbedding, the volume of $U$ in the induced metric is

$$
\begin{aligned}
\operatorname{Vol}_{\Phi^{*} g}(U) & =\int_{R^{n-1} \times(-\varepsilon, \varepsilon)} \sqrt{\operatorname{det}\left(g_{i j}\right)} d x_{1} \cdots x_{n-1} d t \\
& =\int_{R^{n-1} \times(-\varepsilon, \varepsilon)}\left(a(t) \sum_{i=1}^{n-1} x_{i}^{2}+\sum_{i=1}^{n-1} b_{i}(t) x_{i}+c(t)\right) d x_{1} \cdots d x_{n-1} d t \\
& =\infty
\end{aligned}
$$

Since $M$ is compact, any open subset of $M$ should have finite volume. Thus we achieve the needed contradiction which shows that we must have $M_{-}=\varnothing$.

Therefore the null critical metric can only be flat and we complete the proof of Theorem B.

Remark 7.1. As stated in Remark 1.1, a constant curvature metric necessarily has $\mathcal{F}_{2}[g] \geq 0$. Theorem B shows that the converse is true for a critical metric. It should be noted that there indeed exist critical metrics such that $\mathcal{F}_{2}[g]<0$. Take for example, if $n=2 m$, consider the Riemannian product $\left(M^{n}, g\right)=N^{m}(-c) \times S^{m}(c)$, where $c>0$ and $N^{m}(-c)$ denotes a compact space form of constant sectional curvature $-c$, and $S^{m}(c)$ denotes the usual sphere of constant sectional curvature $c$. Now we choose $c$ such that $\operatorname{Vol}\left(M^{n}, g\right)=1$. Then a simple calculation shows that $\left(M^{n}, g\right)$ is a compact, locally conformally flat and non-Einstein manifold with scalar curvature identically zero. By using Corollary 4.1, we can easily prove that $g$ is in fact a critical point of $\left.\mathcal{F}_{2}\right|_{\mathcal{M}_{1}}$ with $\sigma_{2}\left(C_{g}\right)=-m(m-1)^{2} c^{2}<0$.

\section{ACKNOWLEDGEMENTS}

This paper was mainly carried out and completed when both authors were staying at the TU Berlin, with the first author a visiting scholar and the second author an Alexander von Humboldt research fellow. The authors express their thanks to Professor U. Simon for his hospitality and kind support. The first author also expresses his deep gratitude to Professor Viaclovsky who kindly gave very helpful explanations about his joint work with Professor Gursky.

\section{REFERENCES}

[1] A. L. Besse, Einstein Manifolds, Springer-Verlag, Berlin, 1987. MR 88f:53087

[2] J. Cheeger and D. Gromoll, The splitting theorem for manifolds of nonnegative Ricci curvature, J. Diff. Geom., 6(1971/72): 119-128. MR 46:2597

[3] A. Derdzinski, Classification of certain compact Riemannian manifolds with harmonic curvature and non-parallel Ricci tensor, Math. Z., 172(1980): 273-280. MR 82e:53053

[4] M. J. Gursky and J. A. Viaclovsky, A new variational characterization of three-dimensional space forms, Invent. Math., 145(2001): 251-278. MR 2002j:53039

[5] H. Li, Global rigidity theorems of hypersurfaces, Ark. Math., 35(1997): 327-351. MR 98j:53074

[6] A. Lichnerowicz, Propagateurs et commutateurs on relativité générale, Inst. Hautes Études Sci. Publ. Math. No.10(1961), 56. MR 28:967

[7] M. Okumura, Hypersurfaces and a pinching problem on the second fundamental tensor, Amer. J. Math., 96(1974): 207-213. MR 50:5701 
[8] R.C. Reilly, Variational properties of function of the mean curvatures for hypersurfaces in space forms, J. Diff. Geom., 8(1973): 465-477. MR 49:6102

[9] H. Rosenberg, Hypersurfaces of constant curvature in space forms, Bull. Sci. Math., 117(1993): 211-239. MR 94b:53097

[10] J. A. Viaclovsky, Conformal geometry, contact geometry and the calculus of variations, Duke Math. J., 101(2000): 283-316. MR 2001b:53038

[11] K. Voss, Einige differentialgeometrische Kongruenzsätze für geschlossene Flächen und Hyperflächen, Math. Ann., 131(1956): 180-218. MR 18:229f

Department of Mathematics, Zhengzhou University, Zhengzhou 450052, People's RePUBLIC OF CHINA

E-mail address: huzj@zzu.edu.cn

Department of Mathematical Sciences, Tsinghua University, Beijing 100084, People's Republic of China

E-mail address: hli@math.tsinghua.edu.cn 